\title{
Identification of differentially expressed microRNAs in acute Kawasaki disease
}

\author{
YE CHEN $^{1 *}$, YUE-YUE DING $^{1 *}$, YAN REN $^{2}$, LEI CAO $^{1}$, QIU-QIN XU ${ }^{1}$, \\ LING SUN ${ }^{1}$, MING-GUO XU ${ }^{3}$ and HAI-TAO LV $^{1}$ \\ ${ }^{1}$ Cardiology Department, Children's Hospital of Soochow University, Suzhou, Jiangsu 215025; ${ }^{2}$ Radiology Department, \\ Fudan University Affiliated Huashan Hospital, Shanghai 200040; ${ }^{3}$ Cardiovascular Center, \\ Shenzhen Children's Hospital, Shenzhen, Guangdong 518038, P.R. China
}

Received June 11, 2017; Accepted September 27, 2017

DOI: $10.3892 / \mathrm{mmr} .2017 .8016$

\begin{abstract}
The present study used microarray analysis to screen the plasma expression of microRNAs (miRNAs) in patients with acute Kawasaki disease (KD) and aimed to explore the pathogenesis of KD. Plasma was collected from children with acute $\mathrm{KD}(n=6)$ and from healthy control children $(n=6)$. Total RNA was extracted and differential miRNA expression between the two groups was determined. Differentially expressed miRNAs were validated using reverse transcription-quantitative polymerase chain reaction (RT-qPCR) in an independent cohort $(n=8)$. Target genes of the differentially expressed miRNAs were predicted and analyzed for gene ontology term enrichment and Kyoto Encyclopedia of Genes and Genomes pathways. miRNA microarray analysis revealed that seven miRNAs (miRs) were significantly upregulated (hsa-let-7b-5p, hsa-miR-223-3p, hsa-miR-4485, hsa-miR-4644, hsa-miR-4800-5p, hsa-miR-6510-5p and hsa-miR-765) and three were significantly downregulated (hsa-miR-33b-3p, hsa-miR-4443 and hsa-miR-4515) in acute KD compared with the healthy controls. hsa-miR-223-3p expression levels detected by RT-qPCR were consistent with the microarray results. A total of 62 target genes of hsa-miR-223-3p were predicted. In total, 10 differentially expressed miRNAs were identified in acute KD, of which hsa-miR-223-3p was verified by RT-qPCR.
\end{abstract}

\section{Introduction}

Kawasaki disease (KD) is a systemic vasculitis syndrome of unknown etiology. The vasculitis mainly attacks coronary

Correspondence to: Dr Hai-Tao Lv, Cardiology Department, Children's Hospital of Soochow University, 92 Zhongnan Street, Suzhou, Jiangsu 215025, P.R. China

E-mail: haitaosz@163.com

${ }^{*}$ Contributed equally

Key words: Kawasaki disease, microRNAs, microRNA-223-3p, microarray, target gene arteries, and cardiac sequelae, such as coronary aneurysms and coronary insufficiencies, are some of the most serious manifestations of this disease (1-3). It is particularly prevalent in infants and young children (4). Although the clinical features, diagnosis and treatment of KD are well established, its pathogenesis has not been identified yet. Several lines of evidence suggest that an interplay between microbial infection and genetic predisposition serve a role in the development of the disease (5-7).

MicroRNAs (miRNAs) are endogenous single-strand, non-coding RNAs of 18-25 nucleotides in length, that post-transcriptionally regulate gene expression through sequence-specific interaction with target messenger RNAs (mRNAs) $(8,9)$. miRNAs are highly conserved, and their expression is time specific (10). miRNAs exhibit powerful regulatory roles in many biological processes, including cell metabolism, proliferation, differentiation and apoptosis (11). Aberrant expression of miRNAs has been confirmed to be associated with various human diseases including cancers, cardiovascular diseases and inflammatory conditions (12-14). Blood circulating miRNA levels are stable (15) and their unique expression patterns may be used as a novel, non-invasive biomarker for disease diagnosis (16). Recent studies identified circulating miRNAs as biomarkers for many disorders, such as cardiovascular disease $(17,18)$ and inflammatory diseases $(19)$. However, previous reports on the expression of circulating miRNAs in KD are limited. Additional studies are required to determine whether there is differential miRNA expression in the circulating plasma in patients with KD and the functions of target genes.

The present study aimed to identify a panel of plasma miRNAs that are differentially expressed in patients with KD and to provide a possible direction for studying the pathophysiological mechanisms of KD.

\section{Materials and methods}

Specimen source. Plasma specimens for miRNA microarray hybridization were obtained from children with $\mathrm{KD}(\mathrm{n}=6)$ and from healthy control children $(n=6)$ between May 2013 and August 2013; plasma specimens for reverse transcription-quantitative polymerase chain reaction (RT-qPCR) were obtained 
from children with KD $(n=8)$ and from healthy control children $(n=8)$ between September 2013 and October 2013 at the Children's Hospital of Soochow University (Suzhou, China). Patients in the control group underwent regular health examinations and had no infections. KD was defined according to the criteria established by the American Heart Association in 2004 (20). Venous blood (4 ml) was collected from the patients in EDTA-containing tubes on the day of diagnosis for $\mathrm{KD}$. The blood samples were first centrifuged at $820 \mathrm{xg}$ for $10 \mathrm{~min}$ at $4^{\circ} \mathrm{C}$, and then at $16,000 \mathrm{x}$ g for $10 \mathrm{~min}$ at $4^{\circ} \mathrm{C}$. Plasma was collected in $1.5 \mathrm{ml}$ eppendorf tubes and stored at $-80^{\circ} \mathrm{C}$. The 12 biologically independent plasma samples were analyzed individually, rather than pooling the samples. The plasma samples were labeled $\mathrm{K}$ or $\mathrm{C}$ for KD and control, respectively, followed by a coding number, to protect the privacy of the participants during all molecular studies. All parents of participants provided written informed consent for participation in this study, and the samples were processed under the approval of the Ethics Committee of Children's Hospital of Soochow University (Suzhou China).

RNA extraction and quantification. Total RNA was extracted from plasma (400 $\mu \mathrm{l} / \mathrm{sample}$ ) using a mirVana PARIS RNA and Native Protein Purification kit (cat. no. AM1556; Applied Biosystems; Thermo Fisher Scientific, Inc., Waltham, MA, USA), according to the manufacturer's protocol. Briefly, 10 volumes of lysis/binding buffer and 1/10 volume of miRNA homogenate was added to the plasma and mixed well. A 1:1 ratio of acid phenol:chloroform equal to the lysate volume was added to the miRNA homogenate additive. The mixture was centrifuged for $5 \mathrm{~min}$ at $1,000 \mathrm{x}$ g at room temperature, the aqueous upper phase was removed and transferred to a fresh tube. Following the addition of 1.25 volumes $100 \%$ ethanol, the lysate/ethanol mixture was passed through a filter cartridge, which was subsequently washed with $700 \mu \mathrm{l}$ miRNA wash solution 1 and $500 \mu \mathrm{l}$ wash solution 2/3. RNA was eluted from the filters with $100 \mu$ l elution solution that was warmed to $95^{\circ} \mathrm{C}$. Subsequently, the eluate, which contained the RNA, was collected and stored at $-80^{\circ} \mathrm{C}$. Total RNA was quantified using a NanoDrop 2000 spectrophotometer (Thermo Fisher Scientific, Inc.). RNA sample quality was evaluated by an Agilent 2100 Bioanalyzer (Agilent Technologies, Inc., Santa Clara, CA, USA). An RNA $\geq 7.0$ was accepted for microarray analysis and RT-qPCR.

miRNA microarray hybridization. miRNA profile analysis of the plasma samples was performed using a miRNA Microarray Chip V2.4 (Agilent Technologies, Inc.), which contains probes for 2,549 human miRNAs with a sample input of $100 \mathrm{ng}$ total RNA. Briefly, dephosphorylation was performed by gently mixing the total RNA with $2 \mu \mathrm{l}$ calf intestinal alkaline phosphatase master mix (Agilent Technologies, Inc.) and incubating the mixture at $37^{\circ} \mathrm{C}$ in a circulating water bath for $30 \mathrm{~min}$. Subsequently, $2.8 \mu 1100 \%$ dimethyl sulfoxide was added to each sample and incubated at $100^{\circ} \mathrm{C}$ in a circulating water bath for 5-10 min for denaturation. The samples were labeled using a miRNA Complete Labeling and Hybridization kit and hybridized on an Agilent SureHyb Microarray Hybridization Chamber (both from Agilent Technologies, Inc.). Following hybridization, the chip was washed using GE wash buffer 1 and GE wash buffer 2 (Gene Expression Wash Buffer kit; cat. no. 5188-5327; Agilent Technologies, Inc.). The chip was scanned and the data were extracted using Agilent Feature Extraction Software version 10.7.1.1 (Agilent Technologies, Inc.). Data were standardized using GeneSpring Software version 13.1 (Agilent Technologies, Inc.). Fold change $\geq 2$ and $\mathrm{P}<0.05$ were used to indicate significant differences in gene expression, and cluster analysis was performed using Genespring software version 14.8 (Agilent Technologies, Inc.).

$R T-q P C R$. The total RNA extracted from the samples met the quality control requirements and qualified for RT-qPCR analysis. Each $20 \mu \mathrm{l}$ RT reaction was performed according the manufacturer's protocol of the miScript II Reverse Transcriptase kit (Qiagen GmbH, Hilden, Germany) in a GeneAmp PCR system 9700 (Applied Biosystems) for $60 \mathrm{~min}$ at $37^{\circ} \mathrm{C}$, followed by heat inactivation of the RT for $5 \mathrm{~min}$ at $95^{\circ} \mathrm{C}$. qPCR was performed using a LightCycler $480 \mathrm{II}$ (Roche Diagnostics, Basel, Switzerland) with the $10 \mu \mathrm{l}$ reaction mixtures comprising cDNA $(1 \mu \mathrm{l}), 2 \mathrm{X}$ LightCycler 480 SYBR-Green I Master mix (5 $\mu \mathrm{l})$, universal primer $(0.2 \mu \mathrm{l})$, miRNA-specific primer $(0.2 \mu \mathrm{l})$ and nuclease-free water (3.6 $\mu \mathrm{l})$. The upstream primer of hsa-miR-16 was 5'TAG CAGCACGTAAATATTGGCG3'. The upstream primer of hsa-miR-765 was 5'TGGAGGAGAAGGAAGGTGATG3'. The upstream primer of hsa-miR-33b-3p was 5'CAGTGCCTCGGC AGTGCAGCCC3'. The upstream primer of has-miR-223-3p was 5'TGTCAGTTTGTCAAATACCCCA3'. Reactions were incubated in a 384 -well plate at $95^{\circ} \mathrm{C}$ for $10 \mathrm{~min}$, followed by 40 cycles at $95^{\circ} \mathrm{C}$ for $10 \mathrm{sec}$ and at $60^{\circ} \mathrm{C}$ for $30 \mathrm{sec}$. Each sample was run in triplicate. miRNA expression levels were normalized to the internal reference hsa-miR-16 and external reference cel-miR-39 and were determined using the comparative threshold cycle $2^{-\Delta \Delta \mathrm{Cq}}$ method (21).

miRNA target gene prediction. miRNA target genes were predicted using GeneSpring version 13.1 software (Agilent Technologies, Inc.). TargetScan (www.targetscan.org), PITA (genie.weizmann.ac.il/pubs/mir07/mir07_data.html) and microRNA.org (www.microrna.org/microrna/home.do) databases were used to predict the intersectional miRNA target genes. The data were analyzed using Venny software version2.1 (bioinfogp.cnb.csic.es/tools/venny). Common target genes were analyzed for gene ontology (GO) functional term enrichment, such as biological process (BP), cellular component (CC) and molecular function (MF); GeneSpring and Kyoto Encyclopedia of Genes and Genomes (KEGG) were used in the pathway analysis.

Statistical analysis. The patients with KD and control patient sample data were compared using the Wilcoxon rank sum test. Statistical analysis was performed using SPSS version 18.0 (SPSS, Inc., Chicago, IL, USA). $\mathrm{P}<0.05$ was considered to indicate a statistically significant difference.

\section{Results}

Clinical features. Patient samples used for both microarray and RT-qPCR analyses exhibited no differences in sex and age distributions among the patients and controls (Tables I and II). 
Table I. Clinical features of patients with $\mathrm{KD}$ and control patients used in the microarray analysis.

\begin{tabular}{lrrlll}
\hline & \multicolumn{2}{c}{ Age (months) } & & \multicolumn{2}{c}{ Sex } \\
\cline { 2 - 3 } \cline { 6 - 6 } Sample no. & KD & Control & & KD & Control \\
\hline 1 & 25 & 19 & & Female & Male \\
2 & 46 & 17 & & Female & Female \\
3 & 24 & 17 & & Female & Female \\
4 & 8 & 7 & & Male & Male \\
5 & 36 & 37 & & Female & Female \\
6 & 7 & 8 & & Male & Female \\
\hline
\end{tabular}

KD, Kawasaki disease.

Table II. Clinical features of patients with KD and control patients used for reverse transcription-quantitative polymerase chain reaction analysis.

\begin{tabular}{lcclll}
\hline & \multicolumn{2}{c}{ Age (months) } & & \multicolumn{2}{c}{ Sex } \\
\cline { 2 - 3 } Sample no. & KD & Control & & KD & Control \\
\hline 1 & 13 & 15 & & Male & Male \\
2 & 24 & 22 & & Male & Male \\
3 & 34 & 32 & & Male & Female \\
4 & 36 & 31 & & Male & Female \\
5 & 22 & 17 & & Female & Male \\
6 & 11 & 15 & & Female & Male \\
7 & 56 & 49 & & Male & Male \\
8 & 22 & 39 & & Female & Male \\
\hline
\end{tabular}

KD, Kawasaki disease.

Differential miRNA expression. Microarray analysis of the plasma samples from the KD and control groups revealed that seven miRNAs were significantly upregulated (hsa-let-7b-5p, hsa-miR-223-3p, hsa-miR-4485, hsa-miR-4644, hsa-miR-4800-5p, hsa-miR-6510-5p and hsa-miR-765) and three were significantly downregulated (hsa-miR-33b-3p, hsa-miR-4443 and hsa-miR-4515) in the KD plasma samples compared with the control group (Fig. 1; Table III).

$R T-q P C R$. The selected miRNAs hsa-miR-765, hsa-miR-223-3p and hsa-miR-33b-3p underwent RT-qPCR which have been reported in previous studies (22-25) and their target genes can be found in miRNA databases, such as TargetScan (www. targetscan.org) and PITA (genie.weizmann.ac.il/pubs/mir07/ mir07_data.html). Automated RT-qPCR determination of the three miRNAs was performed using hsa-miR-16 as the internal reference. The melting curves indicated good PCR amplification specificity, with one perfect single peak for each miRNA. The relative expression levels of hsa-miR-223-3p and hsa-miR-33b-3p were significantly higher in the KD group compared to the control group ( $\mathrm{P}<0.05$; Fig. 2). The relative expression level of hsa-miR-765 between the two groups was not significantly different $(\mathrm{P}>0.5)$.

Target genes. A total of 62 common target genes of hsa-miR-223-3p were identified by comparing three different target gene predictions and was detected by both RT-qPCR and microarray analysis (Fig. 3 and Table IV).

GO analysis. The 62 predicted target genes of hsa-miR-223-3p were enriched in BPs (including regulation of translation, norepinephrine metabolic process and regulation of neural precursor cell proliferation; Fig. 4A), CCs (including, basolateral plasma membrane, recycling endosome and cytoplasmic vesicle membrane; Fig. 4B) and MFs (including, protein binding, PDZ-domain binding and calmodulin binding; Fig. 4C).

KEGG pathway analysis. The biological pathway enrichment analysis of the 62 predicted target genes showed that hsa-miR-223-3p was significantly enriched in the AMP-activated protein kinase (AMPK) signaling pathway, mineral absorption pathway and signaling pathways regulating pluripotency of stem cell (Fig. 4D).

\section{Discussion}

KD is a childhood multisystemic vasculitis; the mechanisms involved in the pathogenesis of vasculitis are poorly understood. Necrotizing arteritis, subacute chronic vasculitis and luminal myofibroblastic proliferation have been previously identified as the three basic processes of KD pathogenesis (26). Necrotizing arteritis is an acute process that may be responsible for saccular aneurysms. Following the onset of KD, both subacute chronic vasculitis and luminal myofibroblastic proliferation persist for months to years.

The present study identified 10 differentially expressed miRNAs, a number of which have been reported previously, such as hsa-miR-765, hsa-miR-33b-3p and hsa-miR-223-3p. hsa-miR-765 has been reported in coronary disease (22) and cancer (23). hsa-miR-33b-3p has also been reported in cancer (24). And hsa-miR-223-3p has been reported in diabetes mellitus (25), and KD (27). Therefore, RT-qPCR was performed to verify these three miRNAs. As most patients with KD are newborns, the amount of blood that can be withdrawn is limited and that is why different plasma samples were used in microarray analysis and RT-qPCR, which is a limitation of the current study. Given miRNAs strong regulatory roles in cellular metabolism, proliferation, differentiation, apoptosis and stress (reviewed in 11), they may provide clues for understanding the pathophysiology of KD and may be potentially useful in future diagnostic and therapeutic strategies. As they exist in a very stable state in the serum or plasma $(28,29)$, miRNAs are suitable as biological markers for KD diagnosis and follow-up (30). As the results of microarray are not always stable, the present study used RT-qPCR to validate the results of microarray. RT-qPCR validation revealed no difference in hsa-miR-765 expression levels and increased hsa-miR-33b-3p levels in the plasma of acute $\mathrm{KD}$, which was inconsistent with the microarray results. The difference in results between the microarray and RT-qPCR may be due to detection sensitivity 
Table III. Differentially expressed microRNAs of the two groups.

\begin{tabular}{lccllrl}
\hline miRNA & P-value & FC & Trend & Sequence & Chr & miRBase ID \\
\hline hsa-let-7b-5p & $3.15 \times 10^{-2}$ & 8.448197 & Up & AACCACACAACCTACTACC & 22 & MIMAT000003 \\
hsa-miR-223-3p & $3.71 \times 10^{-2}$ & 8.468529 & Up & TGGGGTATTTGACAAACTGAC & X & MIMAT000020 \\
hsa-miR-33b-3p & $4.90 \times 10^{-2}$ & 3.495833 & Down & GGGCTGCACTGCCG & 17 & MIMAT000481 \\
hsa-miR-4443 & $4.26 \times 10^{-2}$ & 2.347132 & Down & AAAACCCACGCCTCC & 3 & MIMAT001891 \\
hsa-miR-4485 & $1.05 \times 10^{-2}$ & 1.699564 & Up & TTAGGGTACCGCGGC & 11 & MIMAT001909 \\
hsa-miR-4515 & $2.21 \times 10^{-2}$ & 2.975258 & Down & GGGCTGCCGGGA & 15 & MIMAT001902 \\
hsa-miR-4644 & $1.00 \times 10^{-7}$ & 34.0109 & Up & CTTCTGTCTCTTTCTCTC & 6 & MIMAT001974 \\
hsa-miR-4800-5p & $4.78 \times 10^{-2}$ & 7.431324 & Up & TCCTTCCTCCTCGG & 4 & MIMAT001998 \\
hsa-miR-6510-5p & $5.53 \times 10^{-3}$ & 2.450577 & Up & GACTCCTCTCTCTCCC & 17 & MIMAT002546 \\
hsa-miR-765 & $4.13 \times 10^{-2}$ & 8.229987 & Up & CATCACCTTCCTTCTCCT & 1 & MIMAT000395
\end{tabular}

Chr, chromosome; miR, microRNA.

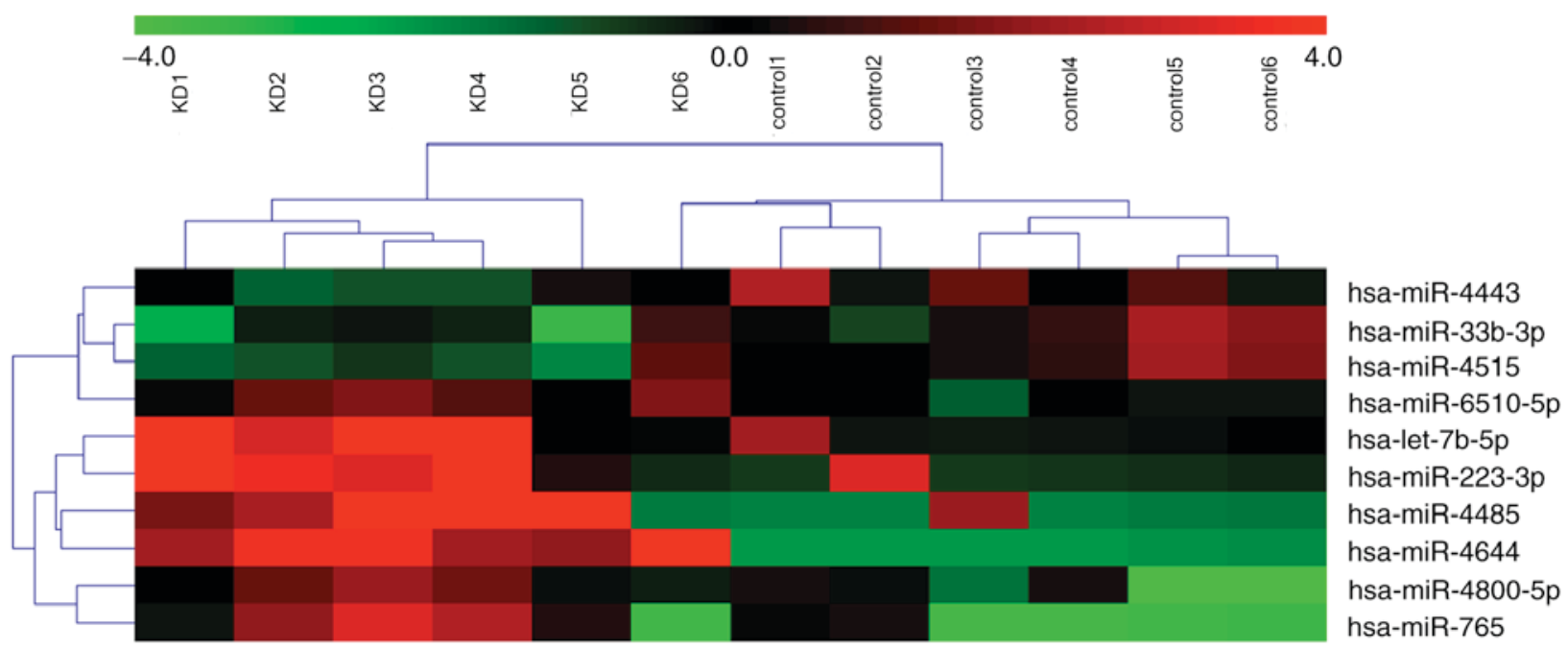

Figure 1. Distinguishable microRNA expression patterns were identified among the samples by hierarchical clustering and displaying by heatmap. In the heatmap, the chromatism represents the level of genes expression, with green corresponding to downregulated and red corresponding to upregulated expression.

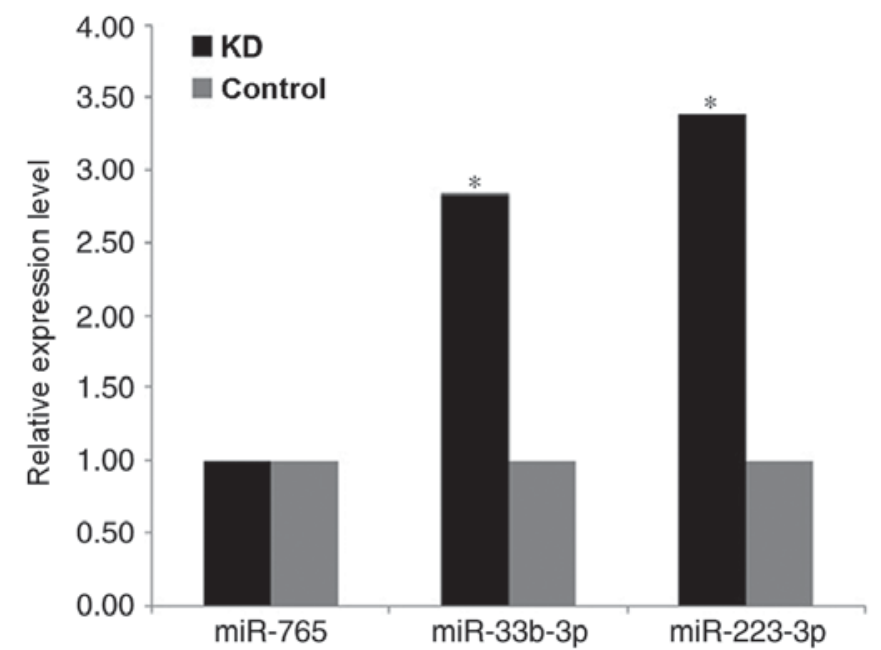

Figure 2. Comparison of the relative expression levels of hsa-miR-765, hsa-miR-223-3p and hsa-miR-33b-3p with the internal reference hsa-miR-16 in the KD and control groups, as measured by reverse transcription-quantitative polymerase chain reaction. ${ }^{*} \mathrm{P}<0.05$ vs. Control. KD, Kawasaki disease; miR, microRNA. differences and sample heterogeneity. In addition, one of the differentially expressed miRNAs, hsa-miR-223-3p, for which both microarray and RT-qPCR revealed increased expression, was selected for target gene prediction.

Currently, few studies $(27,31,32)$ have focused on circulating miRNAs in patients with KD. One previous study used high-throughput sequencing in the peripheral blood in patients with acute and convalescent KD to identify six differentially expressed miRNAs, including miR-143, miR-199b-5p, miR-618, miR-223, miR-145 and miR-145* (27). Using a group of febrile patients with KD as the control, another study reported elevated serum levels of miRNA-200c and miR-371-5p in patients with KD (31). High levels of miR-182 and miR-296-5p have been reported during the acute febrile phase, whereas high levels of miR-93, miR-145*, miR-145 and miR-150-3p were detected in the defervescence stage (32). It has been suggested that miR-93 may regulate vascular endothelial growth factor (VEGF-A) expression and may contribute to the understanding of the pathogenesis of arteritis in acute KD. A recent study demonstrated significantly higher serum 
Table IV. Predicted target genes of hsa-miR-223-3p.

\begin{tabular}{|c|c|c|c|c|c|}
\hline GeneID & Symbol & GeneID & Symbol & GeneID & Symbol \\
\hline 6477 & SIAH1 & 2872 & MKNK2 & 10600 & USP16 \\
\hline 84133 & ZNRF3 & 5997 & RGS2 & 8763 & CD164 \\
\hline 2034 & EPAS1 & 4848 & CNOT2 & 9962 & SLC23A2 \\
\hline 538 & ATP7A & 1080 & CFTR & 57835 & SLC4A5 \\
\hline 10890 & RAB10 & 160518 & DENND5B & 84312 & BRMS1L \\
\hline 6925 & TCF4 & 3836 & KPNA1 & 26118 & WSB1 \\
\hline 84255 & SLC37A3 & 10492 & SYNCRIP & 143098 & MPP7 \\
\hline 255488 & RNF144B & 1010 & CDH12 & 4774 & NFIA \\
\hline 9852 & EPM2AIP1 & 23250 & ATP11A & 463 & ZFHX3 \\
\hline 64145 & RBSN & 6383 & SDC2 & 23220 & DTX4 \\
\hline 92 & ACVR2A & 91860 & CALML4 & 284403 & WDR62 \\
\hline 9472 & AKAP6 & 23435 & TARDBP & 3131 & HLF \\
\hline 55156 & ARMC1 & 29789 & OLA1 & 4628 & MYH10 \\
\hline 27154 & BRPF3 & 214 & ALCAM & 3572 & IL6ST \\
\hline 9882 & TBC1D4 & 55602 & CDKN2AIP & 2309 & FOXO3 \\
\hline 22883 & CLSTN1 & 5898 & RALA & 9868 & TOMM70 \\
\hline 55588 & MED29 & 490 & ATP2B1 & 26269 & FBXO8 \\
\hline 5581 & PRKCE & 125950 & RAVER1 & 5814 & PURB \\
\hline 154796 & AMOT & 11221 & DUSP10 & 51105 & PHF20L1 \\
\hline 2308 & FOXO1 & 8939 & FUBP3 & 5617 & PRL \\
\hline 149018 & LELP1 & 54842 & MFSD6 & & \\
\hline
\end{tabular}

miR, microRNA.

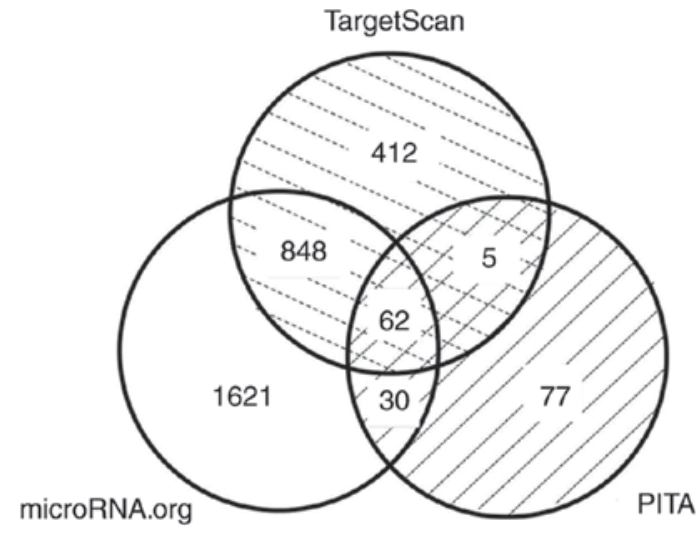

Figure 3. Venn diagram comparing three target-gene predictions by TargetScan, PITA and microRNA.org databases for hsa-miR-223-3p. miR, microRNA.

miR-92a-3p expression levels were detected in children with KD compared with febrile children (33); however, a different study reported that no miRNAs in coronary artery tissues were diagnostic for KD (34). The present study hypothesized that the different sample sources of in vivo circulating blood and in vitro coronary artery tissues contributed to the wholly opposite results. For example, the study by Rowley et al examined miRNA expression in coronary artery tissue from patients who had succumbed to KD (death within weeks after onset), which differed from the study by Rong et al that used circulating blood from living patients with $\operatorname{KD}(33,34)$.
He et al revealed that KD sera suppressed the Krüppel-like factor 4/miR-483 axis in human umbilical vein endothelial cells, and increased the expression of connective tissue growth factor and induction of endothelial-to-mesenchymal transition. This detrimental process in the endothelium may contribute to coronary artery abnormalities in KD patients (35).

Previous studies have demonstrated that miR-223 is expressed in monocytes and macrophages, and may be the key to regulating inflammation (36). miR-223 was also reported to be transported in plasma and delivered to recipient cells by high-density lipoproteins (HDLs) in patients or mice with hypercholesterolemia (37), and it was demonstrated that the anti-inflammatory properties of HDL may be conferred, in part, through HDL-miR-223 delivery and the repression of intercellular adhesion molecule-1 translation in endothelial cells (38). miR-223 was previously demonstrated to target $\beta 1$ integrin to antagonize angiogenesis and prevent growth factor signaling in endothelial cells (39). miR-223 was suggested to be a potential biomarker of type 2 diabetes (25). In addition, platelets were demonstrated to remotely modulate vascular endothelial cell apoptosis by releasing microvesicles that contain miR-223, which targets insulin-like growth factor 1 receptor and promotes advanced glycation end product-induced vascular endothelial cell apoptosis (40). One recent study revealed that high miR-223 expression levels in vascular endothelial cells may function as a novel endocrine genetic signal and participate in vascular injury of KD (41); however, the exact mechanism was not determined. 
A

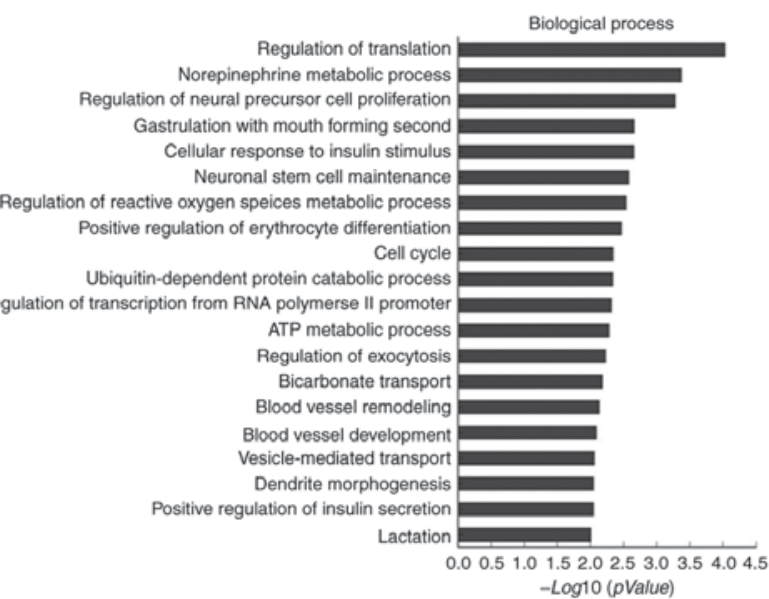

C

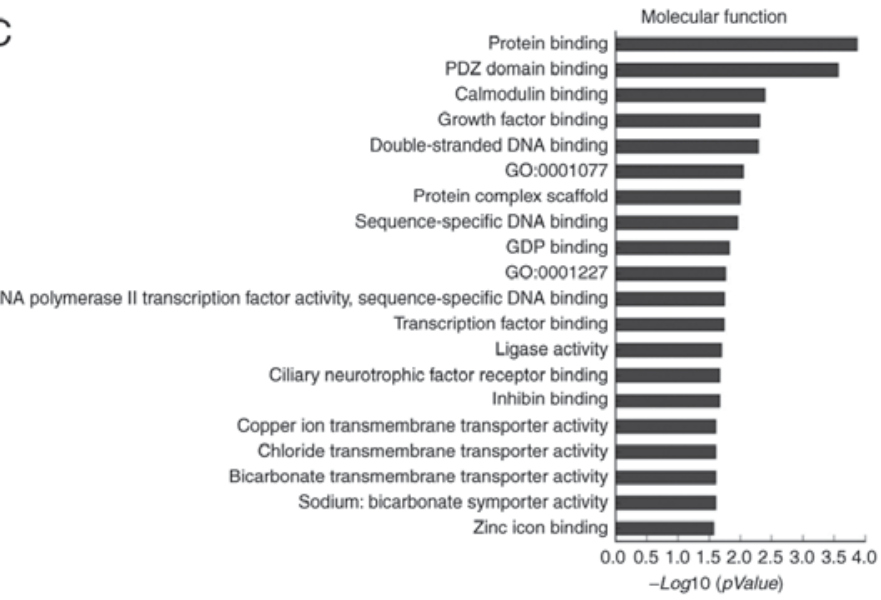

B

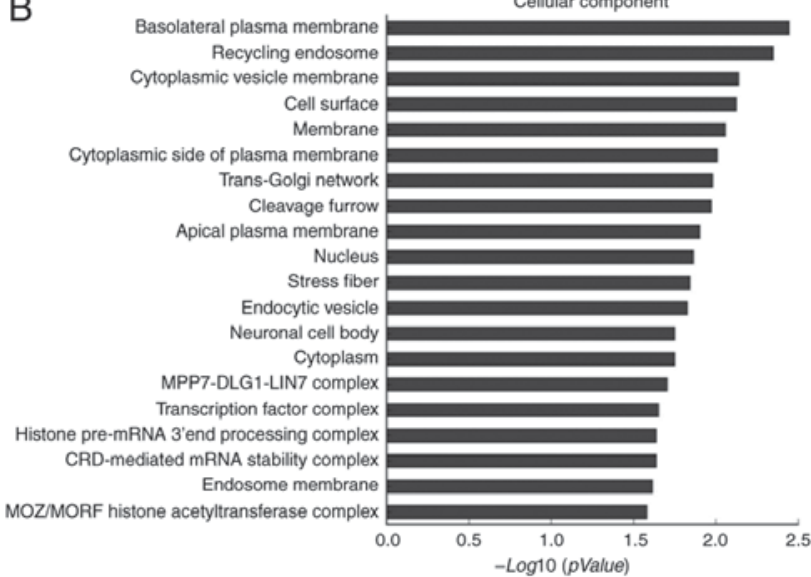

D

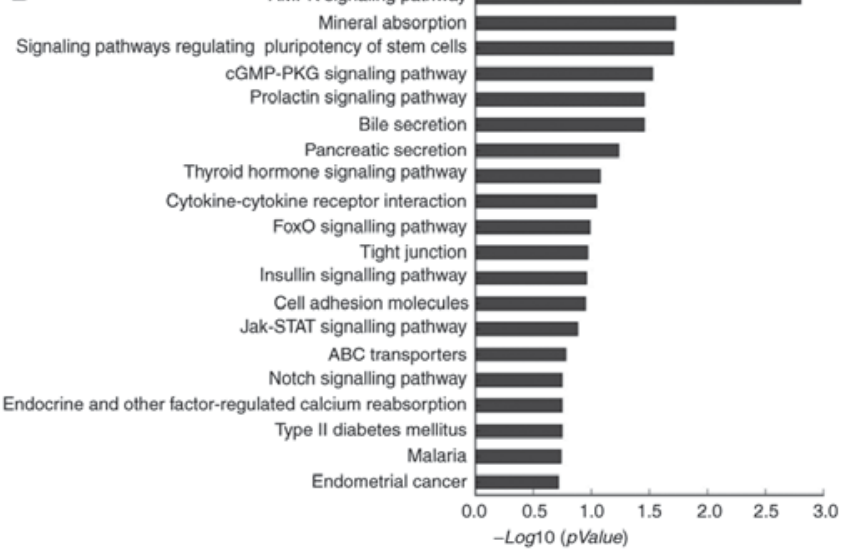

Figure 4. Gene ontology term enrichment and KEGG pathway analysis of the predicted target genes of hsa-miR-223-3p. (A) Enrichment of the biological process of the predicted target genes. (B) Enrichment of the cellular components of the predicted target genes. (C) Enrichment of the molecular functions of the predicted target genes. (D) Enrichment of KEGG pathways of the predicted target genes. KEGG, Kyoto Encyclopedia of Genes and Genomes; miR, microRNA.

The present study identified 62 putative target genes of hsa-miR-223-3p. GO term enrichment analysis identified a number of biological processes, cellular components and molecular functions that may be related to KD; KEGG pathway analysis indicated that the target genes were enriched in AMPK signaling, mineral absorption and signaling pathways regulating stem cell pluripotency, whose role in KD needs to be defined. Previous studies have indicated the existence of specific signals or pathways in KD. For example, it was predicted that, along with other differentially expressed miRNAs, miR-145 may participate in regulating the expression of genes in the transforming growth factor $\beta$ (TGF- $\beta$ ) pathway of arterial wall myofibroblasts (27), and miR-93 may participate in regulating VEGF-A expression in the pathogenesis of arteritis in acute KD (32); signaling pathways regulating stem cell pluripotency and cytokine-cytokine receptor interaction pathways may individually include TGF- $\beta$ and VEGF-A.

In conclusion, 10 differentially expressed miRNAs were detected by microarray chip in the plasma of patients with acute KD, of which 3 miRNAs were verified by RT-qPCR, but only hsa-miR-223-3p was found to be consistently detected by both. A total of 62 potential target genes of hsa-miR-223-3p were identified. Previous studies have reported that miR-223 may regulate inflammation of vascular endothelial cells.
Therefore, hsa-miR-223-3p may participate in the pathogenesis of KD, and determination of its functions and mechanisms in KD require further verification.

\section{Acknowledgements}

This study was financially supported by the National Natural Science Foundation of China (grant nos. 81370217 and 81570455), the Natural Science Foundation of Young (grant nos. 81400222 and 81300124) and the Suzhou Science and Technology Bureau (grant nos. KJXW2014015 and SYS201633).

\section{References}

1. Sudo D, Monobe Y, Yashiro M, Mieno NM, Uehara R, Tsuchiya K, Tsuchiya K, Sonobe T and Nakamura Y: Coronary artery lesions of incomplete Kawasaki disease: A nationwide survey in Japan. Eur J Pediatr 171: 651-656, 2012.

2. Fukazawa R: Long-term prognosis of Kawasaki disease: Increased cardiovascular risk? Curr Opin Pediatr 22: 587-592, 2010.

3. Tsuda E, Abe T and Tamaki W: Acute coronary syndrome in adult patients with coronary artery lesions caused by Kawasaki disease: Review of case reports. Cardiol Young 21: 74-82, 2011.

4. Makino N, Nakamura Y, Yashiro M, Ae R, Tsuboi S, Aoyama Y, Kojo T, Uehara R, Kotani K and Yanagawa H: Descriptive epidemiology of Kawasaki disease in Japan, 2011 2012: From the results of the 22nd nationwide survey. J Epidemiol 25: 239-245, 2015. 
5. Del Principe D, Pietraforte D, Gambardella L, Marchesi A, Tarissi de Jacobis I, Villani A, Malorni W and Straface E: Pathogenetic determinants in Kawasaki disease: The haematological point of view. J Cell Mol Med 21: 632-639, 2017.

6. Shulman ST and Rowley AH: Kawasaki disease: Insights into pathogenesis and approaches to treatment. Nat Rev Rheumatol 11: 475-482, 2015.

7. Yoon KL: Update of genetic susceptibility in patients with Kawasaki disease. Korean J Pediatr 58: 84-88, 2015

8. Sunderland N, Skroblin P, Barwari T, Huntley RP, Lu R, Joshi A, Lovering RC and Mayr M: MicroRNA biomarkers and platelet reactivity: The clot thickens. Circ Res 120: 418-435, 2017.

9. Zhao Y, Song Y, Yao L, Song G and Teng C: Circulating microRNAs: Promising biomarkers involved in several cancers and other diseases. DNA Cell Biol 36: 77-94, 2017.

10. Tijsen AJ, Pinto YM and Creemers EE: Circulating microRNAs as diagnostic biomarkers for cardiovascular diseases. Am J Physiol Heart Circ Physiol 303: H1085-H1095, 2012.

11. Ebert MS and Sharp PA: Roles for microRNAs in conferring robustness to biological processes. Cell 149: 515-524, 2012.

12. Esquela-Kerscher A and Slack FJ: Oncomirs-microRNAs with a role in cancer. Nat Rev Cancer 6: 259-269, 2006.

13. van Rooij E and Olson EN: MicroRNA: Powerful new regulators of heart disease and provocative therapeutic targets. J Clin Invest 117: 2369-2376, 2007.

14. O'Connell RM, Taganov KD, Boldin MP, Cheng G and Baltimore D: MicroRNA-155 is induced during the macrophage inflammatory response. Proc Natl Acad Sci USA 104: 1604-1609, 2007.

15. Mendell JT and Olson EN: MicroRNAs in stress signaling and human disease. Cell 148: 1172-1187, 2012.

16. Reid G, Kirschner MB and van Zandwijk N: Circulating microRNAs: Association with disease and potential use as biomarkers. Crit Rev Oncol Hematol 80: 193-208, 2011.

17. Wang GK, Zhu JQ, Zhang JT, Li Q, Li Y, He J, Qin YW and Jing Q: Circulating microRNA: A novel potential biomarker for early diagnosis of acute myocardial infarction in humans. Eur Heart J 31: 659-666, 2010.

18. Ji X, Takahashi R, Hiura Y, Hirokawa G, Fukushima Y and Iwai N: Plasma miR-208 as a biomarker of myocardial injury. Clin Chem 55: 1944-1949, 2009.

19. Akbas F, Coskunpinar E, Aynaci E, Oltulu Y and Yildiz P: Analysis of serum micro-RNAs as potential biomarker in chronic obstructive pulmonary disease. Exp Lung Res 38: 286-294, 2012

20. Newburger JW, Takahashi M, Gerber MA, Gewitz MH, Tani LY, Burns JC, Shulman ST, Bolger AF, Ferrieri P, Baltimore RS, et al: Diagnosis, treatment, and long-term management of Kawasaki disease: A statement for health professionals from the committee on rheumatic fever, endocarditis, and Kawasaki disease, council on cardiovascular disease in the young, American heart association. Circulation 110: 2747-2771, 2004.

21. Livak KJ and Schmittgen TD: Analysis of relative gene expression data using real-time quantitative PCR and the 2(-Delta Delta C(T)) method. Methods 25: 402-408, 2001.

22. Ali Sheikh MS, Xia K, Li F,Deng X, Salma U, Deng H, Wei Wei L, Yang TL and Peng J: Circulating miR-765 and miR-149: Potential noninvasive diagnostic biomarkers for geriatric coronary artery disease patients. Biomed Res Int 2015: 740301, 2015.

23. Tömböl Z, Eder K, Kovács A, Szabó PM, Kulka J, Likó I, Zalatnai A, Rácz G, Tóth M, Patócs A, et al: MicroRNA expression profiling in benign (sporadic and hereditary) and recurring adrenal pheochromocytomas. Mod Pathol 23: 1583-1595, 2010.

24. Xu N, LiZ, Yu Z Yan F, Liu Y, Lu X and Yang W: MicroRNA-33b suppresses migration and invasion by targeting c-Myc in osteosarcoma cells. PLoS One 9: e115300,2014.

25. Zhu $\mathrm{H}$ and Leung SW: Identification of microRNA biomarkers in type 2 diabetes: A meta-analysis of controlled profiling studies. Diabetologia 58: 900-911, 2015.

26. Orenstein JM, Shulman ST, Fox LM, Baker SC, Takahashi M, Bhatti TR, Russo PA, Mierau GW, de Chadarévian JP, Perlman EJ, et al: Three linked vasculopathic processes characterize Kawasaki disease: A light and transmission electron microscopic study. PLoS One 7: e38998, 2012.
27. Shimizu C, Kim J, Stepanowsky P, Trinh C, Lau HD, Akers JC, Chen C, Kanegaye JT, Tremoulet A, Ohno-Machado L and Burns JC: Differential expression of miR-145 in children with Kawasaki disease. PLoS One 8: e58159, 2013

28. Mitchell PS, Parkin RK, Kroh EM, Fritz BR, Wyman SK, Pogosova-Agadjanyan EL, Peterson A, Noteboom J, O'Briant KC, Allen A, et al: Circulating microRNAs as stable blood-based markers for cancer detection. Proc Natl Acad Sci USA 105 10513-10518, 2015

29. Fichtlscherer S, Zeiher AM and Dimmeler S: Circulating microRNAs: Biomarkers or mediators of cardiovascular diseases? Arterioscler Thromb Vasc Biol 31: 2383-2390, 2011.

30. Gilad S, Meiri E, Yogev Y, Yogev Y, Benjamin S, Lebanony D, Yerushalmi N, Benjamin H, Kushnir M, Cholakh H, et al: Serum microRNAs are promising novel biomarkers. PLoS One 3: e3148, 2008.

31. Yun KW, Lee JY, Yun SW, Lim IS and Choi ES: Elevated serum level of microRNA (miRNA)-200c and miRNA-371-5p in children with Kawasaki disease. Pediatr Cardiol 35: 745-752, 2014.

32. Saito K, Nakaoka H, Takasaki I, Hirono K, Yamamoto S, Kinoshita K, Miyao N, Ibuki K, Ozawa S, Watanabe K, et al: MicroRNA-93 may control vascular endothelial growth factor A in circulating peripheral blood mononuclear cells in acute Kawasaki disease. Pediatr Res 80: 425-432, 2016.

33. Rong X, Jia L, Hong L, Pan L, Xue X, Zhang C, Lu J, Jin Z, Qiu H, Wu R and Chu M: Serum miR-92a-3p as a new potential biomarker for diagnosis of Kawasaki disease with coronary artery lesions. J Cardiovasc Transl Res 10: 1-8, 2017.

34. Rowley AH, Pink AJ, Reindel R, Innocentini N, Baker SC, Shulman ST and Kim KY: A study of cardiovascular miRNA biomarkers for Kawasaki disease. Pediatr Infect Dis J 33: 1296-1299, 2014.

35. He M, Chen Z, Martin M, Zhang J, Sangwung P, Woo B Tremoulet AH, Shimizu C, Jain MK, Burns JC and Shyy JY: miR-483 Targeting of CTGF suppresses endothelial-to-mesenchymal transition: Therapeutic implications in Kawasaki disease. Circ Res 120: 354-365, 2017.

36. Johnnidis JB, Harris MH, Wheeler RT, Stehling-Sun S, Lam MH, Kirak O, Brummelkamp TR, Fleming MD and Camargo FD: Regulation of progenitor cell proliferation and granulocyte function by microRNA-223. Nature 451: 1125-1129, 2008.

37. Vickers KC, Palmisano BT, Shoucri BM, Shamburek RD and Remaley AT: MicroRNAs are transported in plasma and delivered to recipient cells by high-density lipoproteins. Nat Cell Biol 13: 423-433, 2011.

38. Tabet F, Vickers KC, Cuesta Torres LF, Wiese CB, Shoucri BM, Lambert G, Catherinet C, Prado-Lourenco L, Levin MG, Thacker S, et al: HDL-transferred microRNA-223 regulates ICAM-1 expression in endothelial cells. Nat Commun 5: 3292, 2014.

39. Thum T: MicroRNA-223 made its way into vascular research Circ Res 113: 1270-1271, 2013

40. Pan Y, Liang H, Liu H, Li D, Chen X, Li L, Zhang CY and Zen K: Platelet-secreted microRNA-223 promotes endothelial cell apoptosis induced by advanced glycation end products via targeting the insulin-like growth factor 1 receptor. J Immunol 192: 437-446, 2014

41. Chu M, Wu R, Qin S, Hua W, Shan Z, Rong X, Zeng J, Hong L, Sun Y, Liu Y, et al: Bone marrow-derived microRNA-223 works as an endocrine genetic signal in vascular endothelial cells and participates in vascular injury from Kawasaki disease. J Am Heart Assoc 6: pii: e004878, 2017.

This work is licensed under a Creative Common attribution-NonCommercial-NoDerivatives 4.0 International (CC BY-NC-ND 4.0) License. 